r ScIDioc
International Journal of Stem Cell Research and Transplantation (IJST) ISSN: 2328-3548

\section{Paracrine Hypothesis and Cardiac Repair}

Haider $\mathrm{KH}^{1 *}$, Aziz $\mathrm{S}^{2}$

${ }^{1}$ Department of Basic Sciences, Sulaiman AlRajhi Colleges, Kingdom of Saudi Arabia.

${ }^{2}$ George Washington University, M Street NW, Suite, Washington DC, USA.

Keywords: Cytokine; Heart; IL-6; Infarction; Mesenchymal Stem Cells; TGF- $\beta$; TNF- $\alpha$.

Abbreviations: CRP $=$ C-Reactive Protein; HUVEC $=$ Human Umbilical Vein Endothelial Cells; IL-6 = Interleukin-6; Mesenchymal Stem Cells = MSCs; TGF- $\beta=$ Transforming Growth Factor-Beta; TNF $-\alpha=$ Tumor Necrosis Factor - Alpha.

\section{Commentary}

Last two decades of research has seen the emergence and progress of stem cells from a myth to reality and their expediency in the clinical perspective as an effective therapeutic modality. Despite immense progress and promising results from experimental animal studies and clinical trials, the provocative underlying mechanism of their functional efficacy has led to diverging opinions and indulging the researchers into a continuous discussion. With controversies clouding the potential of stem cells to adopt morpho functionally competent cardiac phenotype, paracrine activity of the transplanted stem cells has been put forth as an alternative mechanism associated with the beneficial outcome of cell therapy. Although unique paracrine activity of a cell, besides endocrine activity and juxtacrine activity, constitutes an integral part of the cell-to-cell communication, the release of trophic factors from the transplanted cells favorably modulates the local microenvironment in the cell transplanted region in the infarcted heart and positively impact the integration and reparability of the cell graft. Besides, the donor cells via their paracrine activity provide a conducive microenvironment for the host cardiac cells and enhance their survival via initiation of survival signaling. Additionally, the paracrine trophic factors create a chemical gradient to promote extravasation of bone marrow derived stem/progenitor cells into peripheral circulation for ultimate homing-in to the injured myocardium along with the resident cardiac stem cells to participate in the repair process. The use of paracrine factor-rich conditioned medium has also been used as an adjunct to cell therapy to enhance the engraftment of the donor cells in the heart [1]. Despite wide acceptance of the paracrine hypothesis and publication of a plethora of studies that depict the release of a wide-array of trophic factors by various stem/progenitor cells including the bone marrow derived mesenchymal stem cells (MSCs), there is no single study published as yet that comprehensively profiles their paracrine activity. The secretome is cell-type dependent and is unique for each cell type under a given set of its culture conditions. For example, the composition of secretome of bone marrow derived MSCs cultured under hypoxic conditions varies with the level of oxygen [2]. Besides being affluent in growth factors and cytokines, recent studies have reported the shedding of exosomes as part of their paracrine activity [3]. Exosomes are extracellular vesicles of endocytic origin and with less than $100 \mathrm{~nm}$ diameter and enriched in proteins and microRNAs [4]. Microarray profiling of the exosomes for their contents showed that MSCs use exosomes as carriers to deliver a distinct array of microRNAs to the neighboring cells as part of their mircine activity under given set of conditions [5, 6]. Transplantation of MSCs or injectionbased delivery of their derivative exosomes help in transfer of the microRNAs many of which have significant role in physiological functioning of cardiomyocytes at molecular and cellular levels as well as in the repair of the infarcted myocardium [7].

An important step forward in the exploitation of paracrine hypothesis is the cell-free therapeutic interventional approach wherein conditioned medium in toto or its fractionated components such as growth factors and exosomes from the bone marrow derived progenitor cells is used to treat the ischemic heart. The importance of the cell-free therapy is to exploit the trophic

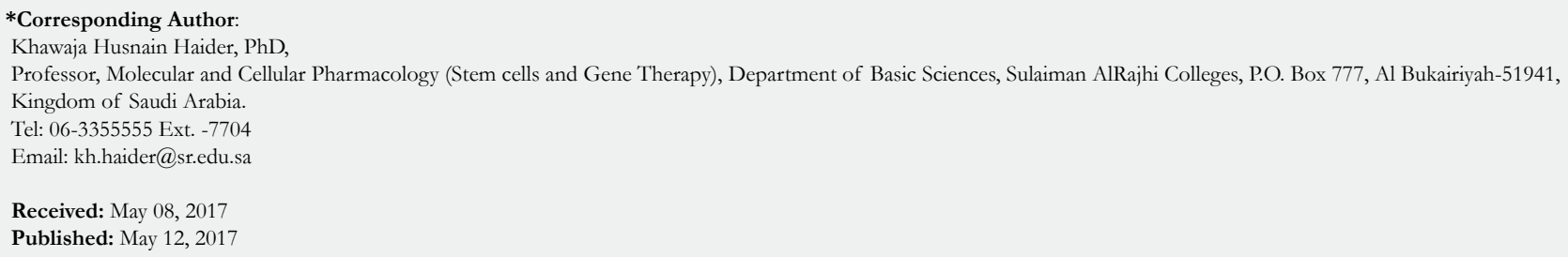


factor-rich conditioned medium that may be directly injected in and around the infarcted heart under direct vision at multiple injection sites [8]. Alternatively, systemic administration of conditioned medium is carried out at multiple stipulated time-points for sustenance of the therapeutic benefits for longer period of time. The promise of cell-free strategy also alleviates the difficulty of optimal cell types, its source and in vitro culture besides immunological rejection of donor cell graft post engraftment. As the conditioned medium can be stored, it's off-the-shelf availability also addresses the logistic concerns associated with the use of cell-based therapy.

The recent study published in IJTS by Amirfarghani et al., (2016) has signified the protective effects of the bone marrow derived MSC conditioned medium in rabbit heart model of experimentally induced myocardial infarction [8]. The model was developed by coronary artery ligation followed by direct intramyocardial injection of either in vitro expanded MSCs or their derivative conditioned medium in the infarcted myocardium. Transthoracic echocardiography at 1,4 and 8 weeks after the respective treatment revealed sustained preservation of left ventricular contractile function and attenuated remodeling of the infarcted heart. The authors have discussed the altered serum levels of Tumor necrosis factor- $\alpha$ (TNF- $\alpha$ ), Interleukin-6 (IL-6) and Tumor growth factor- $\beta$ (TGF- $\beta$ ) in the animals at different time-points after their respective treatment with MSCs or their derivative conditioned medium and have attributed to the beneficial effect of either treatment approach. The observed attenuated expression of TGF- $\beta$ in the study is a desirable feature of the both MSCs as well as conditioned medium treatment as TGF- $\beta$ is involved in each component of the myocardial remodeling after infarction episode including cardiomyocyte apoptosis, fibrogenesis and myocardial hypertrophy [9]. However, the elevated level of TGF- $\beta$ during the early phase of infarction is cardio-protective and protects the heart against early phase inflammatory response [10]. On the contrary, levels of IL-6 show a curved elevation profile earlier on during acute myocardial infarction and correlates well with $\mathrm{C}$-reactive protein (CRP) expression indicating its impact as a mediator of the early phase inflammatory response [11]. Similar to other members of the family, IL-6 imparts its cytoprotective effects through downstream activation of STAT3 with its possible interplay with ERK1/2 in different cell types [12, 13]. For example, pre-treatment of HUVEC with recombinant IL-6 and IL-11 significantly enhanced their survival upon subsequent exposure to oxidative stress [12]. Cardiomyocyte specific knockdown of STAT3 results in higher level of inflammation sensitivity and increase cardiac fibrosis [14]. The cytoprotective effects of the both IL- 6 and IL-11 have been attributed to STAT3 signaling besides the involvement of microRNA-21 [15]. TNF- $\alpha$ is a pro-inflammatory cytokine and a key modulator of any inflammatory response during acute phase myocardial injury [16]. Released by various cell types including macrophages and monocytes, the cardiac fibroblasts and cardiomyocytes have also been implicated as source of TNF- $\alpha$ in the event of ischemic injury to the myocardium with as yet undefined mechanism $[17,18]$. In vitro studies have shown that prolonged exposure of cardiomyocytes activates HIF-1 $\alpha$ dependent signaling in the cells to promote expression of secretion of TNF- $\alpha$ [19]. There is a close interplay between the three cytokines in temporal fashion that oversee the events that follow any infarction episode [20]. A more in-depth mechanistic study would help to understand the importance of regulating their expression using MSC conditioned medium.

Although the data of the published study [8] is interesting, it is not without its limitations. Protein expression level profiling of the conditioned medium for the presence of the three reported cytokines and their expression level changes in the myocardium at stipulated time-points would have been interesting. Similarly, complete profiling of growth factors in the conditioned medium would have immensely added to the impact of study results. Additionally, there is little information about the molecular mechanism by which cell-free conditioned medium from MSCs regulated the interplay between the three cytokines and their time-dependent expression in the event of myocardial infarction. An in-depth mechanistic study is therefore needed to address these issues such that the trio of cytokines can be exploited as possible therapeutic target in the infarcted heart using cell-free conditioned medium of the stem/progenitor cells.

\section{References}

[1]. Rubach M, Adelmann R, Haustein M, Drey F, Pfannkuche K, et al., (2014) Mesenchymal stem cells and their conditioned medium improve integration of purified induced pluripotent stem cell-derived cardiomyocyte clusters into myocardial tissue. Stem Cells Dev. 23(6): 643-653.

[2]. Pawitan AD (2014) Prospect of stem cell conditioned medium in regenerative medicine. BioMed Res Int. 2014: 965849, 14.http://dx.doi. org/101155/2014/965849.

[3]. Lai RC, Chen TS, Lim Sk (2011) Mesenchymal stem cell exosome: a novel stem cell-based therapy for cardiovascular disease. Regen Med. 6(4): 481492.

[4]. Théry C, Zitvogel L, Amigorena S (2002) Exosomes: composition, biogenesis and function. Nature Rev Immunol. 2: 569-579.

[5]. Zomer A, Vendrig T, Hopmans ES, van Eijndhoven M, Middeldorp JM, et al., (2010) Exosomes: Fit to deliver small RNA. Commu Integ Biol. 3(5): 447-450.

[6]. Phinney DG, Giuseppe MD, Njan J, Sala E, Shiva S, et al., (2015) Mesenchymal stem cells use extracellular vesicles to outsource mitophagy and shuttle microRNAs. Nat Commun. 6: 8472 doi: 10.1038/ncomms9472.

[7]. Haider Kh H, Sen KC, Khan M (2015) MicroRNAs with megafunctions cardiac remodeling and repair: the micromanagement of matters of the heart. MicroRNAs in regenerative medicine. 569-600.

[8]. Amirfarhangi A, Dezfouli MG, Abarkar M, Rakhshan K, Aboutaleb N, et al., (2016) Protective role of bone marrow derived mesenchymal stem cellsconditioned medium in the infarcted myocardium: The potential role of selected cytokines. Int J Stem Cell Res Transplant. 4(8): 243-250.

[9]. Euler G (2015) Good and bad sides of TGF $\beta$-signaling in myocardial infarction. Front Physiol. 6: 66.

[10]. Ikeuchi M, Tsutsui H, Shiomi T, Matsusaka H, Matsushima S, et al., (2004) Inhibition of TGF-beta signaling exacerbates early cardiac dysfunction but prevents late remodeling after infarction. Cardiovasc Res. 64(3): 526-535.

[11]. Gabriel AS, Martinsson A, Wretlind B, Ahnve S (2004) IL-6 levels in acute and post myocardial infarction: their relation to CRP levels, infarction size, left ventricular systolic function, and heart failure. Eur J Intern Med. 15(8): 523-528.

[12]. Waxman AB, Mahboubi K, Knickelbein RG, Mantell LL, Manzo N, et al., (2003) Interleukin-11 and interleukin-6 protect cultured human endothelial cells from H2O2- induced cell death. Am J Respir Cell Mol Biol. 29(4): $513-522$.

[13]. Jia Y, Zhou F, Peng Deng P, Qin Fan, Chunhua Li, et al., (2016) Interleukin 6 protects $\mathrm{H} 2 \mathrm{O} 2$-induced cardiomyocytes injury through upregulation of prohibitin via STAT3 phosphorylation. Cell Biochem Function. 30(5): 426-431.

[14]. Jacoby JJ, Kalinowski A, Mu-Gen Liu M-G, Chai GX, Ji L, et al., (2003) Cardiomyocyte-restricted knockout of STAT3 results in higher sensitivity to inflammation, cardiac fibrosis, and heart failure with advanced age. PNAS. 100(22): 12929-12934.

[15]. Haider KhH, Idris NM, Kim HW, Ahmed PH, Shujia J, et al., (2010) MicroRNA-21 is a key determinant in IL-11/Stat3 anti-apoptotic signaling pathway in preconditioning of skeletal myoblasts. Cardiovasc Res. 88(1): 168-178.

[16]. Padfield GJ, Din JN, Koushiappi E, Mills NL, Robinson SD, et al., (2013) Cardiovascular effects of tumor necrosis factor $\alpha$ antagonism in patients 
with acute myocardial infarction: a first in human study. Heart. 99(18): $1330-1335$.

[17]. Tian M, Yuan Y-C, Li J-Y, Gionfriddo MR, Huang R-C (2015) Tumor necrosis factor- $\alpha$ and its role as a mediator in myocardial infarction: A brief review. Chr Dis Transl Med. 1: 18-26.

[18]. Aoyagi T, Matsui T (2011) The cardiomyocytes as a source of cytokines in cardiac injury. J Cell Sci Ther. 2012(0): 003.
[19]. Yu X, Deng L, Wang D, Li N, Chen X, et al., (2012) Mechanism of TNFalpha autocrine effects in hypoxic cardiomyocytes: initiated by hypoxia inducible factor 1alpha, presented by exosomes. J Mol Cell Cardiol. 53(6): 848-857.

[20]. Kempf K, Haltern G, Füth R, Herder C, Martin S, et al., (2006) Increased TNF-alpha and decreased TGF-beta expression in peripheral blood leukocytes after acute myocardial infarction. Horm Metab Res. 38(5): 346-51. 